\title{
Modified fluctuation-dissipation and Einstein relation at nonequilibrium steady states
}

\author{
Debasish Chaudhuri ${ }^{1, *}$ and Abhishek Chaudhuri ${ }^{2,3, \dagger}$ \\ ${ }^{1}$ FOM Institute for Atomic and Molecular Physics, Science Park 104, NL-1098XG Amsterdam, The Netherlands ${ }^{\ddagger}$ \\ ${ }^{2}$ Department of Biomedical Science, University of Sheffield, Western Bank, Sheffield S10 2TN, United Kingdom \\ ${ }^{3}$ The Rudolf Peierls Centre for Theoretical Physics, University of Oxford, 1 Keble Road, Oxford OX1 3NP, United Kingdom
}

(Received 4 November 2011; published 1 February 2012)

\begin{abstract}
Starting from the pioneering work of Agarwal [G. S. Agarwal, Zeitschrift für Physik 252, 25 (1972)], we present a unified derivation of a number of modified fluctuation-dissipation relations (MFDR) that relate response to small perturbations around nonequilibrium steady states to steady-state correlations. Using this formalism we show the equivalence of velocity forms of MFDR derived using continuum Langevin and discrete master equation dynamics. The resulting additive correction to the Einstein relation is exemplified using a flashing ratchet model of molecular motors.
\end{abstract}

DOI: 10.1103/PhysRevE.85.021102

PACS number(s): 05.70.Ln, 05.40.-a, 05.60.-k

\section{INTRODUCTION}

Derived within linear response theory, the fluctuation dissipation theorem (FDT) predicts how the response function of a thermodynamic observable is related to correlation of thermal fluctuations at equilibrium. Let us assume that an equilibrium system described by a Hamiltonian $H$ is perturbed at time $t=t_{1}$ by an external force $h(t)$. The FDT predicts a response at a later time $t_{2}>t_{1}[1]$

$$
R_{A}^{\mathrm{eq}}\left(t_{2}-t_{1}\right)=\frac{\delta\left\langle A\left(t_{2}\right)\right\rangle}{\delta h\left(t_{1}\right)}=\beta \frac{\partial}{\partial t_{1}}\left\langle A\left(t_{2}\right)\left[-\partial_{h} H\left(t_{1}\right)\right]_{h=0}\right\rangle_{\mathrm{eq}}
$$

where the correlation is calculated at equilibrium corresponding to temperature $T$ with $\beta=1 / T$. The differential operator $\partial_{h}$ in the above relation denotes the scalar derivative evaluated at time $t_{1}$. Thus $-\partial_{h} H$ is the displacement conjugate to $h$ with respect to the Hamiltonian. Throughout this paper we use the Boltzman constant $k_{B}=1$, unless otherwise stated. Using the Onsager regression hypothesis the FDT can be interpreted as follows: The decay of a fluctuation is independent of how it has been created, under the influence of a small applied force or spontaneously by thermal noise. The FDT is violated away from the equilibrium regime and this violation has been studied in context of glassy systems, granular matter, sheared fluid, stochastic processes, and biological systems [1-9].

In a pioneering study back in 1972 [10], Agarwal obtained a modified fluctuation-dissipation relation (MFDR) that related response functions around nonequilibrium steady states (NESS) to correlations evaluated at a steady state. For a system evolving with a statistical dynamics characterized by the Fokker-Planck (FP) equation $\partial_{t} p=\mathcal{L}_{0} p$, Agarwal showed that a perturbation in the operator $\mathcal{L}_{0} \rightarrow \mathcal{L}_{0}+h(t) \mathcal{L}_{1}$ leads to a response that can be expressed in terms of a correlation function evaluated at the unperturbed steady state $[10,11]$

$$
R_{A}\left(t_{2}-t_{1}\right)=\frac{\delta\left\langle A\left(t_{2}\right)\right\rangle}{\delta h\left(t_{1}\right)}=\left\langle A\left(t_{2}\right) M\left(t_{1}\right)\right\rangle,
$$

\footnotetext{
*d.chaudhuri@amolf.nl

†a.chaudhuri1@physics.ox.ac.uk

${ }^{\ddagger}$ Current address: Indian Institute of Technology Hyderabad, Yeddumailaram 502205, Andhra Pradesh, India.
}

where the Agarwal term $M=\left[\mathcal{L}_{1} p_{s}\right] / p_{s}$ with $p_{s}$ denoting the steady-state probability distribution. Throughout this paper by $\langle\ldots\rangle$ we denote a steady-state average.

Over the last decade a formalism of stochastic thermodynamics has been developed that allows the description of energy and entropy along fluctuating trajectories [12-14]. Various fluctuation theorems involving the distribution of entropy [15-21] and work theorems [22-24] were discovered. Recently, using an integral fluctuation theorem, a number of these relations were derived in a unified manner [14,25]. Important experimental tests include colloidal particles manipulated by laser traps [26-28], biomolecules pulled by AFM or laser tweezer [29,30], and the autonomous motion of motor proteins [31]. Stochastic thermodynamics has also been used to derive several versions of MFDR around NESS $[6,7,9,32-36]$. Some of these predictions were experimentally verified [27,37].

In this paper, we present a unified derivation of a number of MFDRs based entirely on the Agarwal formalism [10]. Thus the MFDRs we obtain are intrinsically equivalent to each other. We show that the Agarwal term $M$ can be expressed as a velocity excess from a local mean velocity using both the continuиm Langevin and discrete master equation dynamics. This interpretation leads us to a modified Einstein relation that has the same additive correction term for the two cases. Finally, we apply this framework to a flashing ratchet model of molecular motors [38-40] to calculate the MFDR and the additive correction in the Einstein relation, which shows a nonmonotonic variation with the asymmetry parameter of the ratchet.

The structure of this paper is as follows. In Sec. II we review the derivation of the Agarwal form of MFDR that we use throughout this paper to calculate other versions of MFDR expressed in physically observable forms. Using this result, in Sec. III we present a simple and straightforward derivation of the MFDR in terms of stochastic entropy production, keeping in mind that this relation was used earlier to derive velocity MFDR for a master equation dynamics [9]. Then, directly using the Agarwal form, we derive the velocity MFDR for a system evolving with continuum Langevin dynamics in Sec. IV, and a discrete master equation in Sec. V. The velocity MFDR is used in Sec. VI to derive a modified Einstein relation at NESS. In Sec. VII, we study the velocity MFDR, and the 
violation of the Einstein relation in a flashing ratchet model of molecular motors. Finally, in Sec. VIII we summarize our main results and conclude.

\section{THE AGARWAL FORM OF MFDR}

The probability distribution $p(\varsigma, t)$ of finding a system at state $\varsigma$ at time $t$ evolves with time as

$$
\partial_{t} p(\varsigma, t)=\mathcal{L}(\varsigma, h) p(\varsigma, t),
$$

where $\mathcal{L}$ is a general time evolution operator that depends on external force $h(t)$. For weak $h$, Taylor expanding the operator we get

$$
\mathcal{L}(\varsigma, h)=\mathcal{L}_{0}(\varsigma)+h(t) \mathcal{L}_{1}(\varsigma),
$$

where $\mathcal{L}_{1}=\left[\partial_{h} \mathcal{L}\right]_{h=0}$. The solution to Eq. (3) is

$$
p(\varsigma, t)=p_{s}+\int_{-\infty}^{t} d \tau e^{\mathcal{L}_{0}(t-\tau)} h(\tau) \mathcal{L}_{1} p_{s}(\varsigma),
$$

where $p_{s}$ denotes the steady-state distribution that obeys $\mathcal{L}_{0} p_{s}=0$. Then the response of any observable $\langle A(t)\rangle=$ $\int d \varsigma A(\varsigma) p(\varsigma, t)$ to a force $h(t)$ is

$$
\begin{aligned}
R_{A}\left(t_{2}-t_{1}\right) & =\frac{\delta\left\langle A\left(t_{2}\right)\right\rangle}{\delta h\left(t_{1}\right)}=\int d \varsigma A(\varsigma) \frac{\delta p\left(\varsigma, t_{2}\right)}{\delta h\left(t_{1}\right)} \\
& =\int d \varsigma A(\varsigma) e^{\mathcal{L}_{0}\left(t_{2}-t_{1}\right)} \mathcal{L}_{1} p_{s}(\varsigma) \\
& =\int d \varsigma A(\varsigma) e^{\mathcal{L}_{0}\left(t_{2}-t_{1}\right)} M(\varsigma) p_{s}(\varsigma),
\end{aligned}
$$

where in the last step we used the Agarwal term $M(\varsigma) \equiv$ $\left[\mathcal{L}_{1} p_{s}\right] / p_{s}$. By definition, the two-time correlation function is $\langle A(t) B(0)\rangle=\int d \varsigma \int d \varsigma^{\prime} A(\varsigma) B\left(\varsigma^{\prime}\right) p_{2}\left(\varsigma, t ; \varsigma^{\prime}, 0\right)$, where $p_{2}\left(\varsigma, t ; \varsigma^{\prime}, 0\right)$ is the joint probability distribution of finding the system at state $\varsigma^{\prime}$ at time 0 and at state $\zeta$ at time $t$. One can express $p_{2}\left(\varsigma, t ; \varsigma^{\prime}, 0\right)=w\left(\varsigma, t \mid \varsigma^{\prime}, 0\right) p\left(\varsigma^{\prime}, 0\right)$, where $w\left(\varsigma, t \mid \varsigma^{\prime}, 0\right)$ is the transition probability. The time evolution $\partial_{t} p=\mathcal{L}_{0} p$ can be solved to obtain the transition probability at steady state $w\left(\varsigma, t \mid \varsigma^{\prime}, 0\right)=\exp \left(\mathcal{L}_{0} t\right) \delta\left(\varsigma-\varsigma^{\prime}\right)$. Thus the twotime correlation at a steady state takes the form $\langle A(t) B(0)\rangle=$ $\int d \varsigma A(\varsigma) \exp \left(\mathcal{L}_{0} t\right) B(\varsigma) p_{s}(\varsigma)$. Therefore we can write Eq. (6) as

$$
R_{A}\left(t_{2}-t_{1}\right)=\left\langle A\left(t_{2}\right) M\left(t_{1}\right)\right\rangle .
$$

This is the Agarwal form of MFDR [10]. The derivation presented here used a continuum notation of the phase space variable $\varsigma$. However, the result is general, and can be derived similarly for a system that evolves through transitions between discrete states [see Eq. (21)].

The Agarwal term in its operator form $M(\varsigma) \equiv\left[\mathcal{L}_{1} p_{s}\right] / p_{s}$ requires detailed knowledge of the probability distribution at a steady state. In the rest of this paper we focus on expressing this term in physically observable forms.

\section{MFDR IN TERMS OF STOCHASTIC ENTROPY}

The definition of nonequilibrium Gibb's entropy $S=$ $-\int d \varsigma p(\varsigma, t) \ln p(\varsigma, t) \equiv\langle s(t)\rangle$ has recently been used to get a definition of the stochastic entropy $s(t)=-\ln p(\varsigma, t)$ [25]. For a master equation based discrete dynamics between states denoted by $n(t)$, the stochastic entropy can be written as $s(t)=-\ln p_{n(t)}$. Using this definition we obtain a simple interpretation of the Agarwal term in terms of stochastic entropy

$$
M=\frac{1}{p_{s}} \mathcal{L}_{1} p_{s}=\left.\frac{\partial_{h} \mathcal{L}(h) p}{p}\right|_{h=0}=\left.\frac{\partial_{h} \partial_{t} p}{p}\right|_{h=0}=-\partial_{t}\left[\partial_{h} s\right]_{h=0} .
$$

In deriving the above relation we assumed that $\mathcal{L}(h)$ is linear in $h$. We also used the fact that the steady-state distribution $p_{s}=\left.p\right|_{h=0}$. Thus $M$ is expressed as the time evolution of a variable conjugate to the external force $h$ with respect to the stochastic system entropy $s$. In this sense, $s$ in NESS plays a role similar to the Hamiltonian in equilibrium FDT. We can now write the MFDR at NESS as

$$
R_{A}\left(t_{2}-t_{1}\right)=\frac{\partial}{\partial t_{1}}\left\langle A\left(t_{2}\right)\left[-\partial_{h} s\left(t_{1}\right)\right]_{h=0}\right\rangle .
$$

The authors of Refs. [7,41] found this relation by considering a perturbation that takes the system to a final steady state. Note that our simple and straightforward derivation does not require such an assumption, and thus the result is more general.

\section{A. Equilibrium FDT}

The FDT at equilibrium can easily be derived from Eq. (9). If, even in the presence of external perturbation the system remains at equilibrium, one can write down the probability distributions as $p=\exp [-\beta(H-F)]$ where $F$ is the free energy. This distribution leads to the relation $\left[\partial_{h} p\right]_{h=0}=$ $\beta\left[\left(\partial_{h} F-\partial_{h} H\right) p\right]_{h=0}$. Note that the equilibrium displacement evaluated at $h=0$ is $\left[\partial_{h} F\right]_{h=0}=0$. Thus we get the identity $\left[\partial_{h} s\right]_{h=0}=-\left[\left(\partial_{h} p\right) / p\right]_{h=0}=\beta\left[\partial_{h} H\right]_{h=0}$, which leads to the equilibrium FDT Eq. (1).

\section{VELOCITY MFDR USING LANGEVIN EQUATION}

Let us consider a Langevin system where the dynamics of a particle evolves by

$$
v=\mu f+\eta,
$$

where $v=\dot{x}$ is the particle velocity, $\mu$ is the mobility, and $f$ denotes the total force imparted on the particle. The total force $f(x, t)$ consists of a force due to interaction $F(x)$ and an external time-dependent force $h(t): f(x, t)=F(x)+h(t)$. The last term $\eta$ denotes a thermal noise that obeys $\langle\eta\rangle=0$ and $\langle\eta(t) \eta(0)\rangle=2 D \delta(t)$ with $D=\mu T$ the equilibrium Einstein relation. The corresponding FP equation is

$$
\begin{gathered}
\partial_{t} p(x, t)=-\partial_{x} j(x, t), \\
\text { with, } \quad j(x, t)=\left[\mu f(x, t)-D \partial_{x}\right] p(x, t) .
\end{gathered}
$$

The velocity form of MFDR for a Langevin system was originally derived by the authors of Ref. [34]. Here we briefly outline the derivation starting from the Agarwal form. Equation (11) can be expressed as

$$
\partial_{t} p(x, t)=\left(\mathcal{L}_{0}+h(t) \mathcal{L}_{1}\right) p(x, t),
$$

where $\mathcal{L}_{0}=-\partial_{x}(\mu F)+D \partial_{x}^{2}$ and $\mathcal{L}_{1}=-\mu \partial_{x}$. Thus the Agarwal term $M=-\mu\left(\partial_{x} p_{s}\right) / p_{s}$, and $T M=-D\left(\partial_{x} p_{s}\right) / p_{s}$. 
The definition of the steady-state current $j_{s}$ leads to the relation $D \partial_{x} p_{s}=\mu F(x) p_{s}(x)-j_{s}$. Defining a local mean velocity at steady state $v_{s}(x)=j_{s} / p_{s}(x)$ we can then rewrite $T M=-D\left(\partial_{x} p_{s}\right) / p_{s}=v_{s}(x)-\mu F(x)$. In this relation, using the Langevin equation at the initial steady state $(h=0)$, we get $T M=v_{s}-v+\eta$. Thus, the response function

$$
T R_{A}\left(t_{2}-t_{1}\right)=\left\langle A\left(t_{2}\right)\left[v\left(t_{1}\right)-v\left(t_{1}\right)+\eta\left(t_{1}\right)\right]\right\rangle .
$$

Note that in the Langevin equation $\mu h(t)$ and $\eta(t)$ have the same status, and $A(x, t)$ can be regarded as a functional of noise history. Then it can be shown that [34]

$$
T R_{A}\left(t_{2}-t_{1}\right)=D \frac{\delta\left\langle A\left(t_{2}\right)\right\rangle}{\delta \eta\left(t_{1}\right)}=\frac{1}{2}\left\langle A\left(t_{2}\right) \eta\left(t_{1}\right)\right\rangle .
$$

Thus we can write Eq. (12) as

$$
R_{A}\left(t_{2}-t_{1}\right)=\beta\left\langle A\left(t_{2}\right)\left[v\left(t_{1}\right)-v\left(t_{1}\right)\right]\right\rangle .
$$

This is the velocity form of MFDR, which for the velocity response gives

$$
R_{v}\left(t_{2}-t_{1}\right)=\beta\left\langle v\left(t_{2}\right)\left[v\left(t_{1}\right)-v\left(t_{1}\right)\right]\right\rangle .
$$

Note that the steady-state average of $v$ is the same as the mean velocity

$$
\left\langle v_{s}\right\rangle=\int_{-L / 2}^{L / 2} d x p_{s}(x) v_{s}(x)=\mu\langle F\rangle-D\left[p_{s}\right]_{-L / 2}^{L / 2}=\left\langle v_{s}\right\rangle .
$$

The boundary term $\left[p_{s}\right]_{-L / 2}^{L / 2}=0$ either by a periodic boundary condition [34], or by taking the boundaries to infinity where the probabilities vanish. If the system is at equilibrium, $v=0$, and we get back the well-known equilibrium response

$$
R_{v}^{\mathrm{eq}}\left(t_{2}-t_{1}\right)=\beta\left\langle v\left(t_{2}\right) v\left(t_{1}\right)\right\rangle_{\mathrm{eq}} .
$$

Therefore the nonequlibrium MFDR Eq. (15) can be viewed as the equilibrium FDT with an additive correction $-\beta\left\langle v\left(t_{2}\right) v\left(t_{1}\right)\right\rangle$.

It is interesting to note that using Eq. (13), we can arrive at a nonequilibrium MFDR first obtained by the authors of Ref. [32] for continuous Langevin dynamics and subsequently shown to be true for discrete spin variables (as well as for conserved and nonconserved order parameter dynamics) by the authors of Ref. [33]. Defining the position correlation function $C_{x}\left(t_{2}, t_{1}\right)=\left\langle x\left(t_{2}\right) x\left(t_{1}\right)\right\rangle$ and the corresponding response function $2 T R_{x}\left(t_{2}, t_{1}\right)=\left\langle x\left(t_{2}\right) \eta\left(t_{1}\right)\right\rangle$ [using Eq. (13)], we get the modified MFDR

$$
\left(\partial_{t_{1}}-\partial_{t_{2}}\right) C_{x}\left(t_{2}, t_{1}\right)=2 T R_{x}\left(t_{2}, t_{1}\right)+A\left(t_{2}, t_{1}\right),
$$

where $A\left(t_{2}, t_{1}\right)=\left\langle\mu f\left(t_{1}\right) x\left(t_{2}\right)-\mu f\left(t_{2}\right) x\left(t_{1}\right)\right\rangle$ is the so-called asymmetry which vanishes in the presence of time reversal symmetry. Note that causality demands that the response of the system at time $t_{2}$ to a perturbation at time $t_{1}, R_{x}\left(t_{2}, t_{1}\right)$ is nonzero only when $t_{2} \geqslant t_{1}$. Incorporating the time translation invariance and time reversal symmetry restores the equilibrium FDT $T R_{x}\left(t_{2}, t_{1}\right)=\partial_{t_{1}} C_{x}\left(t_{2}, t_{1}\right)$. Also note that the choice of the observable $V$ in Ref. [36] as a one-dimensional (1D) coordinate $x$, reduces the second term on the right-hand side (r.h.s) of Eq. (13) in Ref. [36] to $\left\langle\left(L-L^{*}\right) V(s) Q(t)\right\rangle=$ $\langle 2(j / \rho) \nabla x Q(t)\rangle=\langle v Q\rangle$. Now setting $Q \equiv v$ (velocity), leads Eq. (13) in Ref. [36] to Eq. (14) in our paper, the velocity form of MFDR.

\section{VELOCITY MFDR USING MASTER EQUATION}

We now focus on a master equation system where the time evolution occurs via transitions between discrete states. Following the authors of Ref. [34], we first derive the discrete form of the Agarwal term $M$. Our main contribution in this section is to express $M$ as an excess velocity, and thus arrive at a velocity form of MFDR, similar to the Langevin system.

We begin by considering a set of discrete states $\{n\}$ and write down the corresponding master equation for the probability $p_{m}(t)$ of finding the system in a state $m$ at time $t$

$$
\partial_{t} p_{m}(t)=\sum_{n}\left[w_{n m} p_{n}(t)-w_{m n} p_{m}(t)\right] \equiv \sum_{n} \mathcal{L}_{m n} p_{n}(t),
$$

where $w_{m n}$ represents the transition rate from state $m$ to $n$ and is generally dependent on the external force $h$. The time evolution operator

$$
\mathcal{L}_{m n}=w_{n m}-\delta_{m n} \sum_{k} w_{m k} .
$$

If the external force $h(t)$ acting on the system is weak, Taylor expanding about $h=0$, we get

$$
\mathcal{L}_{m n}(h)=\left(\mathcal{L}_{0}\right)_{m n}+h(t)\left(\mathcal{L}_{1}\right)_{m n} .
$$

In this relation

$$
\left(\mathcal{L}_{1}\right)_{m n}=w_{n m} \alpha_{n m}-\delta_{m n} \sum_{k} w_{m k} \alpha_{m k},
$$

where $\alpha_{m n}=\left[\partial_{h} \ln w_{m n}\right]_{h=0}$ gives the relative change of rates. Note that the system is prepared in a NESS at $h=0$ characterized by the stationary distribution $\left(p_{n}\right)_{s}$. Then Eq. (6) can be expressed in the discrete notation as

$$
R_{A}\left(t_{2}-t_{1}\right)=\sum_{m, n} A_{m}\left[e^{\mathcal{L}_{0}\left(t_{2}-t_{1}\right)} p\left(t_{1}\right)\right]_{m n} M_{n}=\left\langle A\left(t_{2}\right) M\left(t_{1}\right)\right\rangle,
$$

where the Agarwal term is

$$
\begin{aligned}
M_{m} & =\frac{1}{\left(p_{m}\right)_{s}} \sum_{n}\left(\mathcal{L}_{1}\right)_{m n}\left(p_{n}\right)_{s} \\
& =\sum_{n} \frac{\left(p_{n}\right)_{s}}{\left(p_{m}\right)_{s}} w_{n m} \alpha_{n m}-\sum_{n} w_{m n} \alpha_{m n} .
\end{aligned}
$$

Now we use the above relation to derive the velocity form of MFDR. We assume a displacement $d_{m n}$ associated with each transition from state $m$ to $n$. This has the property $d_{m n}=-d_{n m}$ and gives a definition of velocity $v(t)=\sum_{m} \delta\left(t-\tau_{m}\right) d_{m-1, m}$ [42]. A generalized detailed balance in the presence of the external force $h$

$$
\frac{w_{m n}(h)}{w_{n m}(h)}=\frac{w_{m n}(0)}{w_{n m}(0)} \exp \left[\beta h d_{m n}\right]
$$


leads to the following useful relation

$$
\alpha_{m n}-\alpha_{n m}=\beta d_{m n} .
$$

We also utilize the probability current

$$
J_{m n}=p_{m} w_{m n}-p_{n} w_{n m}=-J_{n m} .
$$

Then from Eq. (22) we find the velocity form of the Agwarwal term

$$
\begin{aligned}
M_{m} & =\sum_{n} \frac{\left(p_{n}\right)_{s}}{\left(p_{m}\right)_{s}} w_{n m} \alpha_{n m}-\sum_{n} w_{m n} \alpha_{m n} \\
& =\sum_{n} \frac{\left(p_{n}\right)_{s}}{\left(p_{m}\right)_{s}} w_{n m}\left(\alpha_{m n}+\beta d_{n m}\right)-\sum_{n} w_{m n} \alpha_{m n} \\
& =\beta \sum_{n} \frac{\left(p_{n}\right)_{s}}{\left(p_{m}\right)_{s}} w_{n m} d_{n m}-\sum_{n} \frac{1}{\left(p_{m}\right)_{s}}\left(J_{m n}\right)_{s} \alpha_{m n} \\
& =\beta\left(v_{m}-v_{m}\right),
\end{aligned}
$$

where

$$
v_{m}=\sum_{n} \frac{\left(p_{n}\right)_{s}}{\left(p_{m}\right)_{s}} w_{n m} d_{n m}, \beta v_{m}=\sum_{n} \frac{\left(J_{m n}\right)_{s}}{\left(p_{m}\right)_{s}} \alpha_{m n} .
$$

These relations lead to the velocity form of MFDR

$$
\begin{aligned}
R_{A}\left(t_{2}-t_{1}\right) & =\sum_{m, n} A_{m}\left[e^{\mathcal{L}_{0}\left(t_{2}-t_{1}\right)} p\left(t_{1}\right)\right]_{m n}\left[\beta\left(v_{m}-v_{m}\right)\right] \\
& =\beta\left\langle A\left(t_{2}\right)\left[v\left(t_{1}\right)-v\left(t_{1}\right)\right]\right\rangle .
\end{aligned}
$$

Note that Eq. (28) agrees with the results obtained by the authors of Refs. [7,9]. In particular, the authors of Ref. [9] used the MFDR expressed in terms of stochastic entropy of the system $s$ [Eq. (9)] to obtain Eq. (28). They used the total stochastic entropy $s_{\text {tot }}=s+s_{m}$ where $s_{m}$ is the stochastic entropy of the medium and showed

$$
\begin{aligned}
& \partial_{h} \dot{s}_{m}(t)=\sum_{m} \delta\left(t-\tau_{m}\right) d_{m-1, m} \equiv v(t)=\sum_{m} \delta_{n(t), m} v_{m}, \\
& \partial_{h} \dot{s}_{\text {tot }}(t)=v(t)=\sum_{m} \delta_{n(t), m} v_{m},
\end{aligned}
$$

where $v_{m}$ and $v_{m}$ are given by Eq. (27).

Note the equivalence of Eq. (28) with Eq. (14). Indeed the analogy of $v$ described here with the local mean velocity $[j(x, t) / p(x, t)]$ in the Langevin system becomes even more clear when we compare the steady-state average $\left\langle v_{s}\right\rangle=$ $\sum_{m}\left(p_{m}\right)_{s} v_{m}$ with $\left\langle v_{s}\right\rangle=\sum_{m}\left(p_{m}\right)_{s} v_{m}$ and find

$$
\left\langle v_{s}\right\rangle=T \sum_{m n} J_{m n} \alpha_{m n}=\sum_{m n}\left(p_{n}\right)_{s} w_{n m} d_{n m}=\left\langle v_{s}\right\rangle .
$$

This relation is the same as Eq. (16) obtained for the Langevin system.

For a velocity response Eq. (28) readily leads us to Eq. (15), already obtained in the context of Langevin dynamics. This completes one of the main achievements of this paper; the Agarwal formalism leads to the same form of velocityMFDR for discrete master equation and continuum Langevin dynamics.

\section{EINSTEIN RELATION}

Using the velocity MFDR [Eq. (15) we find the mobility in NESS

$$
\mu_{s}=\int_{0}^{\infty} d \tau R_{v}(\tau)=\beta \int_{0}^{\infty} d \tau\langle v(\tau)[v(0)-v(0)]\rangle .
$$

On the other hand, the diffusion constant in an NESS having mean velocity $\left\langle v_{s}\right\rangle$ is

$$
D_{s}=\int_{0}^{\infty} d \tau\left\langle\left[v(\tau)-\left\langle v_{s}\right\rangle\right]\left[v(0)-\left\langle v_{s}\right\rangle\right]\right\rangle .
$$

Thus the mobility $\mu_{s}$ and diffusion constant $D_{s}$ at NESS do not satisfy the equilibrium Einstein relation (i.e., $D_{s}-T \mu_{s}=$ $I \neq 0$ ). The difference gives us the modification in the Einstein relation in terms of the violation integral

$$
I \equiv D_{s}-T \mu_{s}=\int_{0}^{\infty} d \tau\left[\langle v(\tau) v(0)\rangle-\left\langle v_{s}\right\rangle^{2}\right] .
$$

Since the form of velocity MFDR for the Langevin equation [Eq. (14)] and master equation [Eq. (28)] are the same, we get the same modified Einstein relation for both the cases.

\section{FLASHING RATCHET MODEL OF MOLECULAR MOTORS}

In this section we apply the concepts developed so far in this paper on a specific realization of the flashing ratchet model of molecular motors [38-40,43,44]. In particular, we calculate the velocity MFDR for this model and derive the violation integral of the corresponding nonequilibrium Einstein relation.

A molecular motor (e.g., kinesin) moves along a polymeric track (e.g., microtubule in a strongly fluctuating thermal environment utilizing intrinsic local assymmetry of the track and chemical energy provided by hydrolysis of Adenosine triphosphate (ATP) to Adenosine diphosphate (ADP) and a phosphate). The binding and hydrolyzing of ATP changes the strength of the interaction of the motor with the polymeric track [43]. Thus a simple two-state approximation of the dynamics of motor proteins was proposed $[43,44]$ where the motor encounters a locally asymmetric but globally periodic potential whose height switches between a large and a small value.

We consider a flashing-ratchet model where the system switches between two states, (1) on state: stochastic motion in an asymmetric piecewise linear potential, (2) off state: simple 1D diffusion (Fig. 1). The probability distributions in the two states $p_{1,2}(x, t)$ evolve by [44]

$$
\begin{aligned}
& \partial_{t} p_{1}+\partial_{x} j_{1}=\omega_{2} p_{2}-\omega_{1} p_{1}, \\
& \partial_{t} p_{2}+\partial_{x} j_{2}=-\omega_{2} p_{2}+\omega_{1} p_{1},
\end{aligned}
$$

where $j_{1}=-D \partial_{x} p_{1}$ and $j_{2}=-D\left[p_{2} \partial_{x}(W / T)+\partial_{x} p_{2}\right]$, and $\omega_{1,2}$ denote the transition rates. In the on state, the potential $W(x)$ is periodic $W(x)=W(x+\lambda)$ with period $\lambda=(a+$ $b)$. Within one period, $W(x)=\left(W_{0} / a\right) x$ if $0 \leqslant x<a$, and $W(x)=\left(W_{0} / b\right)(\lambda-x)$ if $a \leqslant x<\lambda$.

We perform molecular dynamics simulations of a particle moving under the influence of the above-mentioned ratchet potential in the presence of a Langevin heat bath. We use stochastic switching between the on and off states with a constant switching rate $\omega_{1}=\omega_{2}$. From this simulation, in 


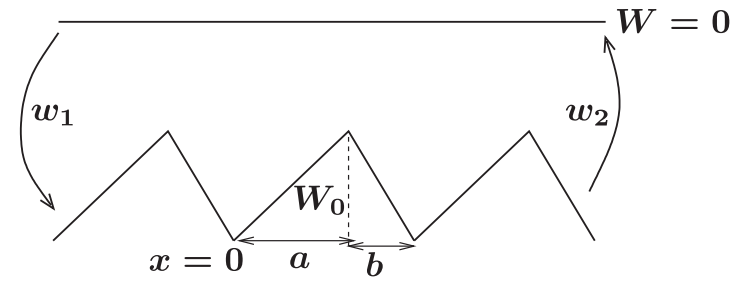

FIG. 1. Flashing ratchet model: The potential height switches between $W=0$ (off state) and $W_{0}$ (on state). The asymmetry of the potential in the on state is described by the inequality $a \neq b . \omega_{1,2}$ denote transition rates between on and off states.

Fig. 2, we plot the velocity-response function $k_{B} T R_{v}(\tau)$ and the related steady-state correlations $\langle v(\tau) v(0)\rangle,\langle v(\tau) v(0)\rangle$ [Eq. (15)]. The parameter values we use are enlisted in Fig. 2 and are typical of microtubule associated molecular motors [45]. At long time, both $\langle v(\tau) v(0)\rangle$ and $\langle v(\tau) v(0)\rangle$ decorrelate to $\left\langle v_{s}\right\rangle^{2}$. We utilize the correlation functions to determine the mobility $\mu_{s}$, diffusion constant $D_{s}$, and the violation integral $I=D_{s}-k_{B} T \mu_{s}$ (see Fig. 2).

We calculate the dependence of the steady-state mobility $k_{B} T \mu_{s}$, diffusion constant $D_{s}$, and the violation integral $I$ on the asymmetry parameter $\alpha=a / \lambda$ (Fig. 3) where $\alpha=1 / 2$ denotes the symmetric ratchet. This calculation leads us to the curious result that all three quantities have a minimum at $\alpha=1 / 2$. The steady-state diffusion constant $D_{s}$ in the flashing ratchet is always suppressed $\left(D_{s}<D\right)$, and moves closer to the free diffusion $D$ for the most asymmetric ratchet. Note that the violation integral quantifies the difference between

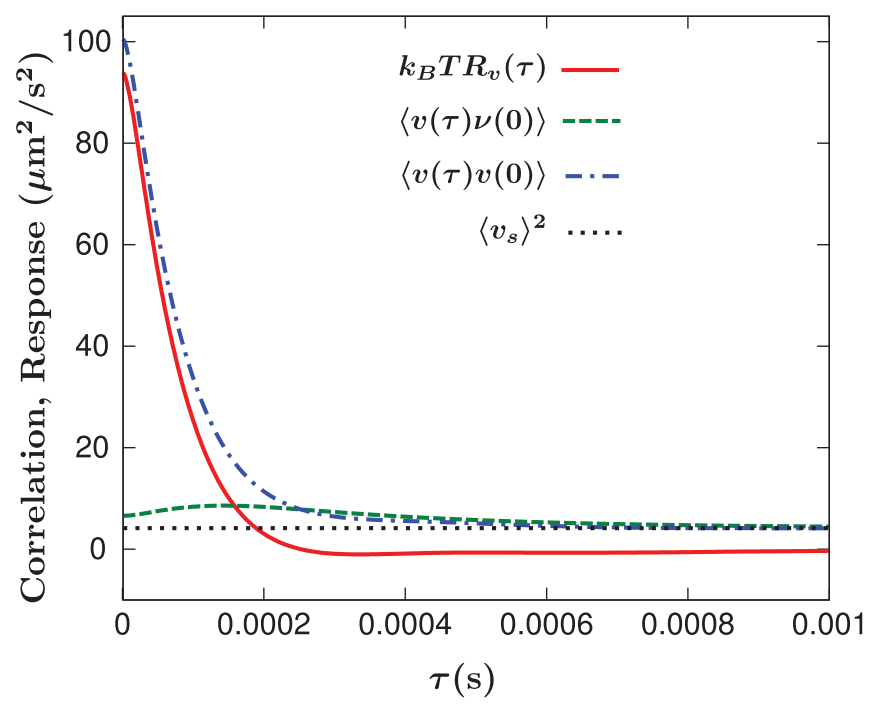

FIG. 2. (Color online) Velocity correlations and response: velocity response $k_{B} T R_{v}(\tau)$, correlation function of the velocity with the local mean velocity $\langle v(\tau) v(0)\rangle$, and velocity autocorrelation function $\langle v(\tau) v(0)\rangle$ as a function of time. The parameter values used to obtain these curves are $\lambda=8 \mathrm{~nm}, a / \lambda=0.1, D=0.009 \mu \mathrm{m}^{2} \mathrm{~s}^{-1}$, $k_{B} T=4.2 \mathrm{pN} \mathrm{nm}, W_{0}=18.85 k_{B} T$, and simulation time step $\delta t=$ $1.8 \times 10^{-6} \mathrm{~s}$. The transition rates are chosen to be equal with $w_{1}=w_{2}=3536 \mathrm{~s}^{-1}$. With these parameter values we find $D_{s}=$ $0.0084 \mu \mathrm{m}^{2} \mathrm{~s}^{-1}, k_{B} T \mu_{s}=0.0057 \mu \mathrm{m}^{2} \mathrm{~s}^{-1}$, and the violation integral $I=0.0027 \mu \mathrm{m}^{2} \mathrm{~s}^{-1}$. The mean velocity in a steady state is $\left\langle v_{s}\right\rangle=$ $2.04 \mu \mathrm{m} / \mathrm{s}$.

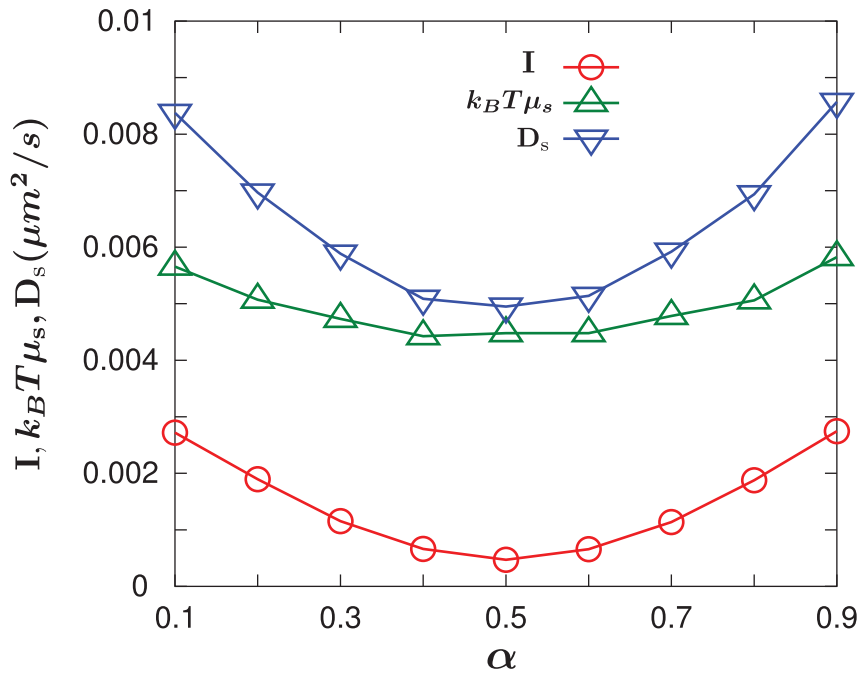

FIG. 3. (Color online) Flashing ratchet: diffusion constant $D_{s}$, mobility $k_{B} T \mu_{s}$, and violation integral $I$ as a function of asymmetry parameter $\alpha=a / \lambda$. All the other parameter values are the same as in Fig. 2.

NESS and equilibrium, with equilibrium requiring $I=0$. The symmetric ratchet does not generate a unidirectional motion, but the switching between the on and off states keeps the system out of equilibrium. Thus, though the violation integral reaches its minimum at $\alpha=1 / 2$ it remains $I \neq 0$. Setting the switching rates $\omega_{1}, \omega_{2}=0$ would restore equilibrium with $I=0$. The dependence of $I$ on various models and parameter values at different NESS is yet to be fully understood.

While the calculation of all the other quantities from our simulations are straightforward, $v(t)$ demands a special mention. The local mean velocity $v(x)$ is the stochastic particle velocity $v$ averaged over the subset of trajectories passing through $x$. At a steady state, this definition is the same as $v_{s}(x)=j_{s} / p_{s}(x)$ where the mean current is constant everywhere: $j_{s}=\rho\left\langle v_{s}\right\rangle$ with $\left\langle v_{s}\right\rangle$ the mean velocity at steady state and $\rho=1 / \lambda$ the mean density. In calculating $\langle v(\tau) \nu(0)\rangle$, the local mean velocity at time $t$ is obtained by identifying the value of $v(x)$ corresponding to the position $x$ visited by the particle at that instant.

\section{SUMMARY}

We have presented a unified derivation of MFDRs at NESS using the Agarwal formalism. Thus all the various versions of MFDR that we derived in this paper are intrinsically equivalent to each other. We showed that the response function around any NESS can be expressed as a correlation between the observable and a variable conjugate to the external force with respect to the system's stochastic entropy production. For both a continuum Langevin and a discrete master equation system, we have shown that the nonequilibrium form of FDT involving velocity response can be expressed as an equilibrium one and an additive correction. The correction in both these cases is a correlation function of the velocity with a local mean velocity. The resulting modification of the Einstein's relation gives the violation in terms of a time integral over this additive correction. 
Using molecular dynamics simulations in the presence of a Langevin heat bath, we studied a flashing ratchet model within this framework and obtained the response function and velocity correlations in the steady state. We showed that the violation integral varies nonmonotonically with the asymmetry parameter of the ratchet and reaches a nonzero minimum for the case of a symmetric ratchet. We plan to extend our study to other models of molecular motors [40], stochastic particle-pumps [46,47], polymer translocation dynamics [48], and dynamics of self-propelled particles [49].

\section{ACKNOWLEDGMENTS}

DC thanks Bela Mulder for useful comments and a critical reading of the manuscript. The work of DC is part of the research program of the "Stichting voor Fundamenteel Onderzoek der Materie (FOM)," which is financially supported by the "Nederlandse organisatie voor Wetenschappelijk Onderzoek (NWO)." AC acknowledges support by Grant No. EP/G062137/1 from the Engineering and Physical Sciences Research Council, UK.
[1] U. Marconi, A. Puglisi, L. Rondoni, and A. Vulpiani, Phys. Rep. 461, 111 (2008).

[2] P. Hänggi and H. Thomas, Phys. Rep. 88, 207 (1982).

[3] P. Martin, A. J. Hudspeth, and F. Jülicher, Proc. Natl. Acad. Sci. USA 98, 14380 (2001).

[4] A. Crisanti and F. Ritort, J. Phys. A: Math. Gen. 36, R181 (2003).

[5] T. Speck and U. Seifert, Phys. Rev. E 79, 040102(R) (2009).

[6] J. Prost, J.-F. Joanny, and J. M. R. Parrondo, Phys. Rev. Lett. 103, 090601 (2009).

[7] U. Seifert and T. Speck, Europhys. Lett. 89, 10007 (2010).

[8] A. Sarracino, D. Villamaina, G. Gradenigo, and A. Puglisi, Europhys. Lett. 92, 34001 (2010).

[9] G. Verley, K. Mallick, and D. Lacoste, Europhys. Lett. 93, 10002 (2011).

[10] G. S. Agarwal, Z. Phys. 252, 25 (1972).

[11] H. Risken, The Fokker-Planck Equation: Methods of Solutions and Applications (Springer-Verlag, Berlin, 1989).

[12] K. Sekimoto, Prog. Theor. Phys. Suppl. 130, 17 (1998).

[13] C. Bustamante, J. Liphardt, and F. Ritort, Phys. Today 58, 43 (2005).

[14] U. Seifert, Eur. Phys. J. B 64, 423 (2008).

[15] D. J. Evans, E. G. D. Cohen, and G. P. Morriss, Phys. Rev. Lett. 71, 2401 (1993).

[16] G. Gallavotti and E. G. D. Cohen, Phys. Rev. Lett. 74, 2694 (1995).

[17] J. L. Lebowitz and H. Spohn, J. Stat. Phys. 95, 333 (1999).

[18] T. Hatano and S.-I. Sasa, Phys. Rev. Lett. 86, 3463 (2001).

[19] D. J. Evans and D. J. Searles, Adv. Phys. 51, 1529 (2002).

[20] M. Esposito, U. Harbola, and S. Mukamel, Phys. Rev. E 76, 031132 (2007).

[21] M. Esposito and C. Van den Broeck, Phys. Rev. Lett. 104, 090601 (2010).

[22] C. Jarzynski, Phys. Rev. Lett. 78, 2690 (1997).

[23] G. E. Crooks, Phys. Rev. E 61, 2361 (2000).

[24] R. Kawai, J. M. R. Parrondo, and C. Van den Broeck, Phys. Rev. Lett. 98, 080602 (2007).

[25] U. Seifert, Phys. Rev. Lett. 95, 040602 (2005).

[26] G. M. Wang, E. M. Sevick, E. Mittag, D. J. Searles, and D. J. Evans, Phys. Rev. Lett. 89, 050601 (2002).
[27] V. Blickle, T. Speck, L. Helden, U. Seifert, and C. Bechinger, Phys. Rev. Lett. 96, 070603 (2006).

[28] D. Andrieux, P. Gaspard, S. Ciliberto, N. Garnier, S. Joubaud, and A. Petrosyan, Phys. Rev. Lett. 98, 150601 (2007).

[29] J. Liphardt, S. Dumont, S. B. Smith, I. Tinoco, and C. Bustamante, Science 296, 1832 (2002).

[30] D. Collin, F. Ritort, C. Jarzynski, S. B. Smith, I. Tinoco, and C. Bustamante, Nature (London) 437, 231 (2005).

[31] K. Hayashi, H. Ueno, R. Iino, and H. Noji, Phys. Rev. Lett. 104, 218103 (2010).

[32] L. Cugliandolo, J. Kurchan, and G. Parisi, J. Phys. I 4, 1641 (1994).

[33] E. Lippiello, F. Corberi, and Marco Zannetti, Phys. Rev. E 71, 036104 (2005).

[34] T. Speck and U. Seifert, Europhys. Lett. 74, 391 (2006).

[35] R. Chetrite, G. Falkovich, and K. Gawedzki, J. Stat. Mech. (2008) P08005.

[36] M. Baiesi, C. Maes, and B. Wynants, Phys. Rev. Lett. 103, 010602 (2009).

[37] J. R. Gomez-Solano, A. Petrosyan, S. Ciliberto, R. Chetrite, and K. Gawedzki, Phys. Rev. Lett. 103, 040601 (2009).

[38] F. Jülicher, A. Ajdari, and J. Prost, Rev. Mod. Phys. 69, 1269 (1997).

[39] R. D. Astumian and P. Hänggi, Phys. Today 55, 33 (2002).

[40] A. B. Kolomeisky and M. E. Fisher, Annu. Rev. Phys. Chem. 58, 675 (2007).

[41] T. Speck, Prog. Theor. Phys. Suppl. 184, 248 (2010).

[42] U. Seifert, Phys. Rev. Lett. 104, 138101 (2010).

[43] R. Astumian and M. Bier, Phys. Rev. Lett. 72, 1766 (1994).

[44] J. Prost, J. F. Chauwin, L. Peliti, and A. Ajdari, Phys. Rev. Lett. 72, 2652 (1994).

[45] H. Wang, C. S. Peskin, and T. C. Elston, J. Theor. Biol. 221, 491 (2003).

[46] K. Jain, R. Marathe, A. Chaudhuri, and A. Dhar, Phys. Rev. Lett. 99, 190601 (2007).

[47] D. Chaudhuri and A. Dhar, Europhys. Lett. 94, 30006 (2011).

[48] M. Muthukumar, J. Chem. Phys. 111, 10371 (1999).

[49] T. Vicsek and A. Zafiris, e-print arXiv:1010.5017. 